\title{
Remote Patient Monitoring Program for Hospital Discharged COVID-19 Patients
}

\author{
William J. Gordon ${ }^{1,2,3}$ Daniel Henderson ${ }^{3,4}$ Avital DeSharone ${ }^{4}$ Herrick N. Fisher ${ }^{1,3}$ Jessica Judge ${ }^{1}$ \\ David M. Levine ${ }^{1,3}$ Laura MacLean $^{5}$ Diane Sousa ${ }^{5}$ Mack Y. Su ${ }^{3}$ Robert Boxer $r^{1,3}$
}

\footnotetext{
${ }^{1}$ Division of General Internal Medicine, Brigham and Women's Hospital, Boston, Massachusetts, United States

2 Department of Medicine, Harvard Medical School, Boston, Massachusetts, United States

${ }^{3}$ Harvard Medical School, Boston, Massachusetts, United States

${ }^{4}$ Department of Medicine, Massachusetts General Hospital, Boston, Massachusetts, United States

${ }^{5}$ Brigham and Women's Hospital, Boston, Massachusetts, United States

Appl Clin Inform 2020;11:792-801.
}

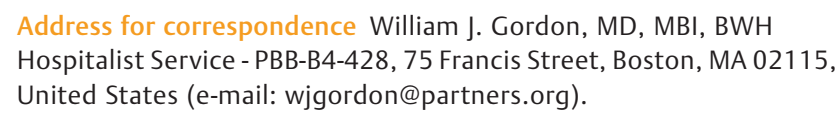

Address for correspondence William J. Gordon, MD, MBI, BWH Hospitalist Service - PBB-B4-428, 75 Francis Street, Boston, MA 02115, United States (e-mail: wjgordon@partners.org).

\author{
Abstract \\ Keywords \\ - remote patient \\ monitoring \\ - coronavirus disease \\ 2019 \\ - patient outcome \\ assessment \\ - remote sensing \\ technology
}

Objective We deployed a Remote Patient Monitoring (RPM) program to monitor patients with coronavirus disease 2019 (COVID-19) upon hospital discharge. We describe the patient characteristics, program characteristics, and clinical outcomes of patients in our RPM program.

Methods We enrolled COVID-19 patients being discharged home from the hospital. Enrolled patients had an app, and were provided with a pulse oximeter and thermometer. Patients self-reported symptoms, $\mathrm{O}_{2}$ saturation, and temperature daily. Abnormal symptoms or vital signs were flagged and assessed by a pool of nurses. Descriptive statistics were used to describe patient and program characteristics. A mixed-effects logistic regression model was used to determine the odds of a combined endpoint of emergency department (ED) or hospital readmission.

Results A total of 295 patients were referred for RPM from five participating hospitals, and 225 patients were enrolled. A majority of enrolled patients (66\%) completed the monitoring period without triggering an abnormal alert. Enrollment was associated with a decreased odds of ED or hospital readmission (adjusted odds ratio: 0.54; 95\% confidence interval: $0.3-0.97 ; p=0.039)$. Referral without enrollment was not associated with a reduced odds of ED or hospital readmission.

Conclusion RPM for COVID-19 provides a mechanism to monitor patients in their home environment and reduce hospital utilization. Our work suggests that RPM reduces readmissions for patients with COVID-19 and provides scalable remote monitoring capabilities upon hospital discharge. RPM for postdischarge patients with COVID-19 was associated with a decreased risk of readmission to the ED or hospital, and provided a scalable mechanism to monitor patients in their home environment. received

August 11, 2020

accepted after revision

October 7, 2020 (c) 2020 Georg Thieme Verlag KG Stuttgart · New York
DOI https://doi.org/

10.1055/s-0040-1721039.

ISSN 1869-0327. 


\section{Background and Significance}

The novel coronavirus "severe acute respiratory syndrome coronavirus 2"-which first emerged in Wuhan, China in late 2019 and caused the respiratory illness coronavirus disease 2019 (COVID-19)-has infected millions worldwide, caused unprecedented morbidity and mortality, and overwhelmed many healthcare delivery systems. ${ }^{1-4}$ Hospitals have been forced to fundamentally shift clinical operations, with canceled procedures, repurposed intensive care units (ICUs), and even staff reductions. ${ }^{5-9}$ Meanwhile, the COVID-19 pandemic has forced an expansion in the use of virtual care, radically accelerating a shift in care delivery that had already begun, now enabled by U.S. regulatory changes and improved pathways to reimbursement. ${ }^{10-14}$

Remote patient monitoring (RPM) is a mechanism for assessing patients outside a typical clinical encounter, for example, in their homes or communities. RPM programs use various telecommunication channels to collect health datasuch as a symptom surveys, vital signs, or data from wearable sensors-and transmit these data to a healthcare provider in a different location. ${ }^{15-19}$ RPM is an attractive care delivery strategy with promise for lower costs, improved convenience, closer monitoring capabilities, and potentially better outcomes. However, data supporting the use of RPM have so far been mixed, with variable results depending on condition and implementation. ${ }^{16,20-26}$

As COVID-19 cases began to emerge in our region, we identified a need for managing an increasing number of patients admitted to the hospital with COVID-19 in the setting of unparalleled resource constraints. We therefore rapidly designed and deployed an RPM program so that we could monitor and manage patients after discharge in their home environment using pulse oximeters, thermometers, and an app-based symptom assessment tool, monitored by a team of nurses.

We identified several potential benefits from such an RPM program for patients with COVID-19. First, most patients with COVID-19 ultimately recover, even among hospitalized populations, and there is a great need for continuity of care posthospitalization. ${ }^{27,28}$ Second, because of the infectious nature of the disease, allowing people to remain in their home could decrease the chance of spread to other patients or staff within the hospital setting. Third, COVID-19 takes a variable clinical course ${ }^{29}$ and identifying patients who may clinically worsen after a hospitalization could have important patient safety benefits. Fourth, an RPM program might allow a discharging clinician to feel more comfortable with an earlier discharge, knowing that the patient would be closely monitored after leaving the hospital, thus freeing up additional inpatient capacity. Finally, as COVID-19 rapidly spread in our geographic region, there was significant provider and patient anxiety around its clinical course, epidemiologic characteristics, and outcomes. By providing a visible layer of perceived security, an RPM program could potentially assuage these concerns.

Numerous institutions have implemented RPM programs in response to COVID-19, and initial work has described
COVID-19 related RPM programs with a focus on implementation, population characteristics, postdischarge, and cohort isolation settings. ${ }^{15,30-36}$ We describe here the characteristics of our patients and the efficacy of this program in the remote management of patients recently admitted with COVID-19.

\section{Methods}

\section{Setting}

MassGeneral Brigham (formerly Partners HealthCare) is a large not-for-profit healthcare system based in Boston, Massachusetts founded by Massachusetts General Hospital and Brigham and Women's Hospital. As of this writing, Massachusetts has had over 125,000 cases of COVID-19 and over 9,000 deaths, ${ }^{37}$ making Massachusetts one of the hardest-hit states. Our program was launched at our two largest hospitals (Massachusetts General Hospital and Brigham and Women's Hospital) during a pilot period, followed by three additional hospitals several weeks later (Newton-Wellesley Hospital, Brigham and Women's Faulkner Hospital, and North Shore Medical Center).

\section{Tool}

We deployed MyChart Care Companion, a module embedded in our patient portal software (Epic Systems Inc., Verona, Wisconsin, United States). Care Companion has both mobile and desktop version, and was available in English and Spanish. The mobile version reminds a patient each morning to complete a survey, at which point the patient is able to self-enter their device data (oxygen saturation and temperature), and answer five symptom questions related to shortness of breath, cough, appetite, weakness, and vomiting. The content was developed by Cleveland Clinic and Epic Systems, Inc. ${ }^{30}$ There were no major technical issues with care companion during the study period.

\section{Study Population and Program Eligibility}

Patients were eligible for the program if they had a diagnosis of COVID-19 (or presumed COVID-19 if nasopharyngeal polymerase chain reaction (PCR) was negative, but clinical suspicion was high, for example, with characteristic symptoms and computed tomography findings), were able to activate a patient portal account, were being discharged home (with or without nursing services), and were able to fill out the survey in English or Spanish (either alone or with the help of a proxy). Exclusion criteria included age $<18$, comorbid highly symptomatic non-COVID-19 conditions outside the scope of RPM triage providers (e.g., advanced heart failure with dyspnea), cognitive or behavioral health barriers to participation that could not be overcome with caregiver support, conditions limiting the ability to work with the devices, lack of a working phone, or discharge to a facility (e.g., skilled nursing facility, short-term rehabilitation, or field hospital).

Eligible patients were referred to the program at the time of discharge through an order in our electronic health record (EHR) and provided with a pulse oximeter (Masimo MightSat 
or the Sensogram Sensoscan), a thermometer (Care Line Inc., oral), an instructional packet on the program, and a selfaddressed stamped envelope for eventual return of the pulse oximeter. These devices were chosen based on extant knowledge about the clinical course of COVID-19 at the time, as well as the availability of devices within our supply chains. Eligible patients who were not already users of our patient portal were guided through the portal enrollment process. All materials were provided free of charge to the patient.

On the day after discharge, all referred patients were contacted by a nurse from our central call center. Patients who were reachable and were able to confirm that they had the app installed (or could access their desktop computer) were considered enrolled. The contacting nurse would also provide assistance for patients or proxies who were having difficulty with the app as needed. Enrolled patients were subsequently prompted through an automated alert or email to complete the questionnaire on the app every morning. If the patient had no concerning symptoms and their objective data were within defined parameters $\left(\mathrm{O}_{2}\right.$ saturation greater than or equal to $92 \%$, temperature less than $100.4 \mathrm{~F}$ ), then no further steps were taken. A message would be sent to a pooled EHR inbox under three conditions: first, if the patient reported worsening symptoms in the questionnaire; second, if their self-entered $\mathrm{O}_{2}$ saturation was less than $92 \%$ or their temperature was greater than 100.3F; and third, if an assigned monitoring task had not been completed in 24 hours. The pooled EHR inbox was staffed by a team of triage nurses 8 A.M. to 8 P.M. in a centralized call-center established specifically for calls related to COVID-19. The nurses would then contact the patient, perform a clinical assessment, and then determine an appropriate plan, for example, refer to the ED, contact the primary care physician, offer empiric treatment, or simply continue monitoring. Given the potential severity of COVID-19 and that our program was started during the early stages of the pandemic, we did not opt for automatic monitoring.

The program was staffed with 24/7 physician backup, and patients were instructed how to reach the on-call physician in the evening hours of 8 P.M. to 8 A.M. when there was no nursing coverage available. After 2 weeks, patients were given the option to complete monitoring or to continue for a third week at patient discretion (-Fig. 1 for detailed enrollment and monitoring workflow).

\section{Statistical Analysis}

Our overall study population included all patients with COVID-19 (or presumed COVID-19) discharged home from one of the five participating hospitals. We split this population into three groups: (1) patients who were not referred to the program, (2) patients who were referred, but not successfully enrolled on post-discharge day 1 , and (3) patients ordered for the program and successfully enrolled on postdischarge day 1 . Group 1 served as our control group. We performed descriptive statistics for each of these groups. We assessed program engagement by analyzing survey responses, length of time in the program, and symptom patterns across the submitted questionnaires. We examined the frequency of questionnaire responses per patient, segmented by whether the questionnaire triggered an alert to the central nursing pool.

A mixed-effects multivariable logistic regression model was used to compute odds ratios (ORs) with 95\% confidence intervals (CIs) for the odds that a patient with COVID-19 who was discharged home from one of our participating hospitals would be readmitted to the emergency department (ED) or the hospital within 30 days of the initial discharge. Covariates included age, ethnicity, language, race (for all groups with at least five readmission events), length of stay of index admission, whether the patient was in an ICU at any point during their admission, income quartile (estimated by zip code), and whether the patient was ordered for the remote monitoring program but not ultimately enrolled, or ordered for the program and enrolled on discharge day 1 (i.e., Group 2 or Group 3 above). Our model included a random-effect term to account for the discharging hospital. COVID-19 status was determined by having an ICD-10 billing code matching COVID.

Data analysis was conducted using R statistical software version 3.5.1 (R Project for Statistical Computing, Vienna, Austria). As a retrospective study on a quality improvement project, the MassGeneral Brigham institutional review board exempted this study from review.

\section{Results}

A total of 1,356 patients with COVID-19 were discharged from one of the five hospitals participating in the RPM program during the study period (April 8, 2020 to June 10, 2020 when the program ended due to declining COVID-19 admissions), 295 were referred for RPM by a discharging provider, and 225 patients were enrolled on post-hospital day 1 . Our two largest hospitals launched first; the remaining three hospitals launched a few weeks later, and 181 patients completed either 14 or 21 days, while 44 were enrolled, but did not end up completing 14 or 21 days of monitoring ( - Fig. 2). - Table 1 shows the baseline characteristics of COVID-19 patients not referred to the program, referred but not ultimately enrolled, or referred and successfully enrolled in the program.

During the study period, 210 patients completed at least one questionnaire, with a total of 2,291 patient days in the program and 2,161 total questionnaires completed. Among patients who completed at least one questionnaire, engagement was high, with a median program duration of 12 days (interquartile range [IQR]: 10-13 days) and a median of 11 (IQR: 8-13) questionnaires completed per patient. The most common symptom that triggered an alert was temperature greater than 100.3 , present in $11 \%$ (239/2161) of all questionnaires, and $76 \%(239 / 315)$ of those leading to an alert (-Table 2).

A majority of patients were monitored without intervention for their entire postdischarge monitoring period. Of the 210 who completed at least one questionnaire, only 72/210 (34\%) triggered a symptom alert to the central nursing pool during their monitoring enrollment period, and only $15 \%$ (315/2161) of questionnaires across all patients triggered an 


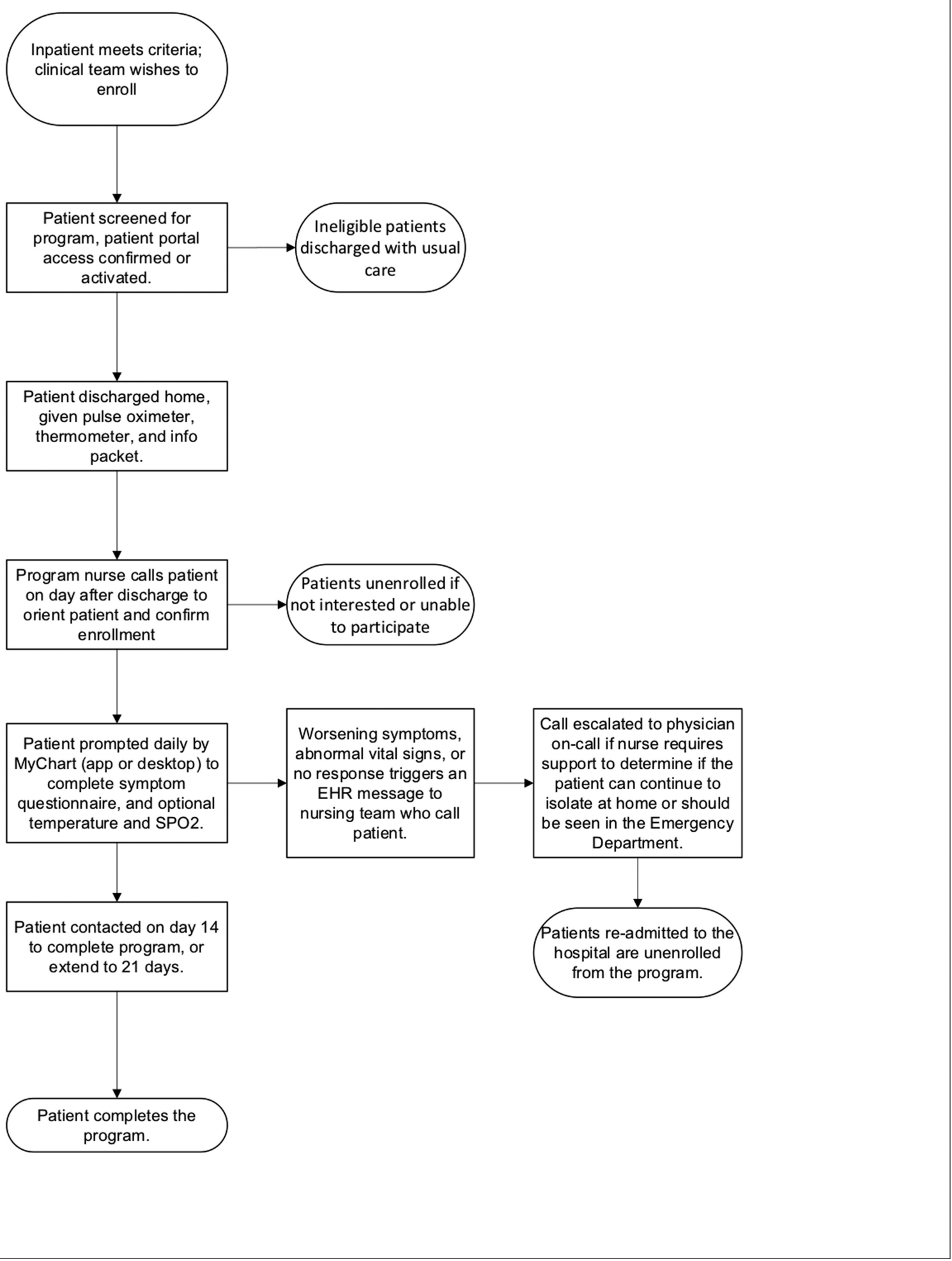

Fig. 1 Detailed enrollment workflow for patients with COVID-19 being discharged from one of the five participating hospitals with the Remote Patient Monitoring program.

alert to the central nursing pool ( - Table 2). - Fig. 3 shows the number of questionnaires completed by program day, split by whether the questionnaire resulted in an alert or escalation to the nursing pool. There was a steady decline in the number of questionnaires per program day over time.
In a multivariable model, we found that being successfully enrolled in the RPM program on postdischarge day 1 was associated with a decreased odds of the combined endpoint of presentation to the ED or readmission (adjusted odds ratio [OR]: 0.54; 95\% CI: 0.3-0.97; $p=0.039$ ), but being ordered for 


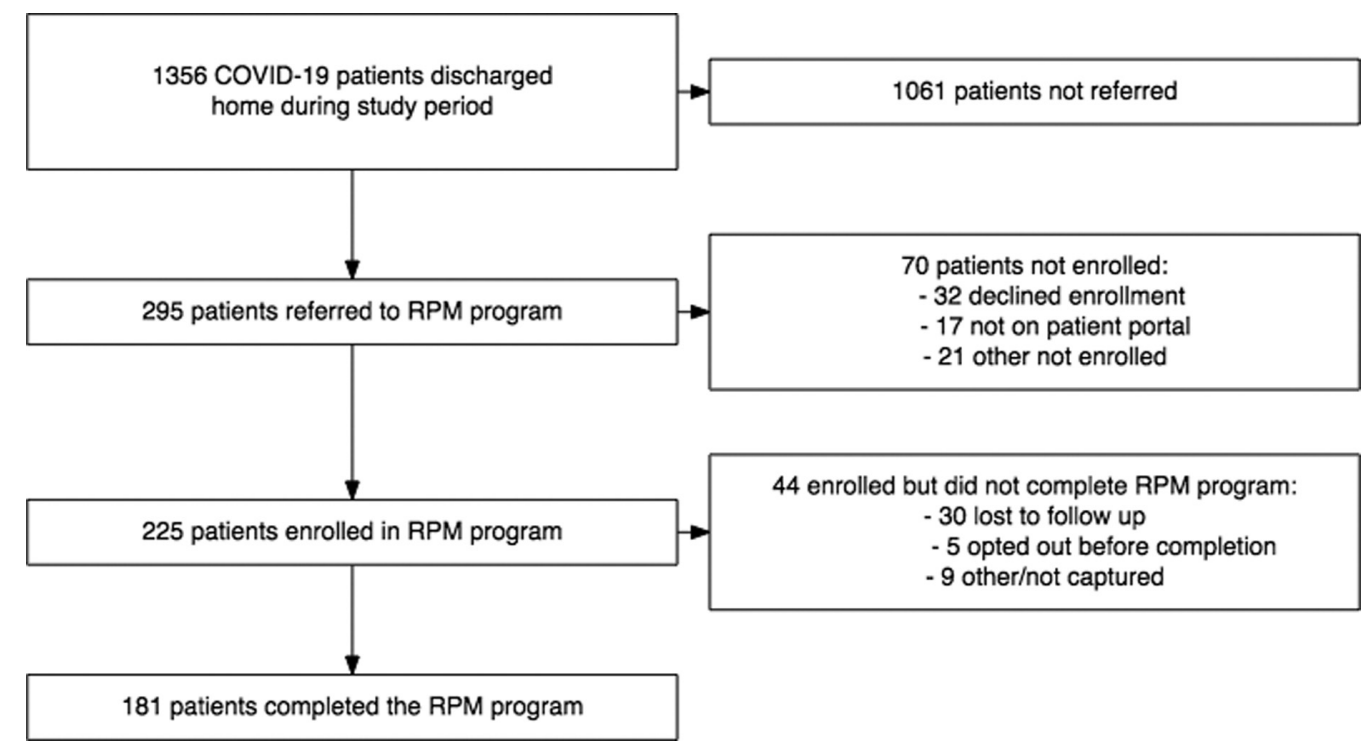

Fig. 2 Remote Patient Monitoring enrollment funnel.

the RPM program without a successful enrollment was not associated with a decreased odds of the combined endpoint (adjusted OR: $1.11 ; 95 \% \mathrm{CI}: 0.51-2.41 ; p=0.793$ ). There were no important readmission associations for other demographic variables such as age, sex, race, ethnicity, or language. Full results are listed in - Table 3 .

\section{Discussion}

We describe the implementation and impact of a posthospitalization portal-based RPM program for patients with COVID-19. We found that the RPM program was associated with a significantly decreased risk of our combined endpoint of ED or hospital readmission for patients who were referred to the program and enrolled on postdischarge day 1. Furthermore, among patients who completed at least one questionnaire while enrolled in the program (210 patients out of 225 enrolled), 66\% did not product a symptom alert that needed manual follow-up, suggesting that RPM for discharged patients with COVID-19 can provide broad monitoring without the need to directly contact patients after hospital discharge beyond the initial enrollment call. This is an important finding because it demonstrates a scalable mechanism for monitoring patients with high risk for clinical worsening without requiring one-to-one engagement.

COVID-19 has forced healthcare systems around the world to innovate and adapt to unprecedented operational and clinical strain. In many ways, the particular challenges presented by the COVID-19 pandemic create a variety of needs for which RPM is uniquely well suited. First, RPM offers the potential to partially ameliorate capacity constraints. By reducing the risk of undetected decompensation after discharge, RPM has the potential to reduce length of stay for medically stable patients who might otherwise get another day of observation. With many hospitals operating well above capacity, the value of even a small reduction in occupancy would be meaningful. Second, RPM may allow health systems to make better use of human resources. Many health systems have redeployed providers to assist with COVID-19 follow-up calls to ensure adequate surveillance for decompensations. In our experience, these calls are often done by prescriber-level providers, and typically take between 8 and 15 minutes. By automating a significant share of time-intensive phone calls, RPM may decrease the cost of COVID-19 follow-up and increase the capacity of providers to provide other higher-value forms of care.

Third, by offering an extra layer of monitoring, relative to usual care, RPM may increase safety and enhance the patient experience. The significant heterogeneity observed in COVID-19 patient trajectories represents a focus of clinical risk and significant worry for patients, their families, and their medical providers. The variable and frequently prolonged clinical course of COVID-19 in particular make RPM appealing, as median symptom duration is heterogeneous and many patients take weeks to recover. ${ }^{29,38}$ Fortunately, most patients have mild disease and do not require hospitalization or escalated care. However, some patients develop moderate or severe disease, and RPM enables a healthcare system to monitor a large set of patients to ensure that the patients that do require escalated care are rapidly identified. Fourth, by reducing direct patient contact, RPM may help reduce the spread of a highly contagious illness, keeping infectious patients in their homes instead of clinical settings (although this could also cause spread to family members). Fifth, as we demonstrate, RPM may have the potential to reduce readmissions to the hospital or ED. We hypothesize that this may be due to the patients having a reliable point of contact, as well as vital sign monitoring for reassurance. Because of significant anxiety around COVID-19, particularly early in the pandemic, in our experience patients had a low threshold for presenting to the ED or hospital, and it is possible that our program led to fewer unnecessary presentations. Finally, by embedding real-time knowledge 
Table 1 Demographics of discharged patients with COVID-19 not ordered for Remote Patient Monitoring, ordered for Remote Patient Monitoring (but not successfully enrolled), and patients ordered as well as successfully enrolled

\begin{tabular}{|c|c|c|c|c|}
\hline Characteristic & $\begin{array}{l}\text { RPM not ordered, } \\
n=1,061^{\mathrm{a}}\end{array}$ & $\begin{array}{l}\text { RPM ordered } \\
\text { (not enrolled), } n=70^{\mathrm{a}}\end{array}$ & $\begin{array}{l}\text { RPM ordered } \\
\text { ( enrolled), } n=225^{\mathrm{a}}\end{array}$ & $p$-Value ${ }^{b}$ \\
\hline Gender (\%) & & & & 0.7 \\
\hline Female & $536(51)$ & $32(46)$ & $114(51)$ & \\
\hline Male & 525 (49) & $38(54)$ & $111(49)$ & \\
\hline Age & $55(41-66)$ & $56(41-69)$ & $54(41-65)$ & 0.6 \\
\hline Language (\%) & & & & 0.028 \\
\hline English & $567(53)$ & $41(59)$ & $142(63)$ & \\
\hline Spanish & $386(36)$ & $24(34)$ & $65(29)$ & \\
\hline Not available/other & $108(10)$ & $5(7.1)$ & $18(8.0)$ & \\
\hline Race (\%) & & & & 0.14 \\
\hline $\begin{array}{l}\text { Black or African } \\
\text { American }\end{array}$ & $179(17)$ & $12(17)$ & $50(22)$ & \\
\hline White & $396(37)$ & $26(37)$ & $85(38)$ & \\
\hline Not available/other & $486(46)$ & $32(46)$ & $90(40)$ & \\
\hline Ethnicity (\%) & & & & 0.4 \\
\hline Hispanic or Latino & $426(40)$ & $31(44)$ & $81(36)$ & \\
\hline Not Hispanic or Latino & $544(51)$ & $35(50)$ & $126(56)$ & \\
\hline Other/not available & $91(8.6)$ & $4(5.7)$ & $18(8.0)$ & \\
\hline Length of stay & $5.0(3.0-8.0)$ & $5.0(2.0-10.0)$ & $5.0(3.0-8.0)$ & 0.4 \\
\hline Required ICU (\%) & $255(24)$ & $23(33)$ & $67(30)$ & 0.068 \\
\hline $\begin{array}{l}\text { Readmission status } \\
(\%)\end{array}$ & & & & 0.15 \\
\hline Not readmitted & $954(90)$ & $62(89)$ & $211(94)$ & \\
\hline Readmitted to ED & $46(4.3)$ & $7(10)$ & $11(4.9)$ & \\
\hline $\begin{array}{l}\text { Readmitted to } \\
\text { hospital }\end{array}$ & $61(5.7)$ & $1(1.4)$ & $3(1.3)$ & \\
\hline $\begin{array}{l}\text { Median income by zip } \\
\text { code }(\%)\end{array}$ & & & & 0.066 \\
\hline $4,836-41,406.5$ & $515(49)$ & $30(43)$ & $88(39)$ & \\
\hline $41,406.5-51,897$ & $272(26)$ & $20(29)$ & $72(32)$ & \\
\hline $51,897-65,903.5$ & $169(16)$ & $12(17)$ & $37(16)$ & \\
\hline $65,903.5-244,671$ & $99(9.3)$ & $8(11)$ & $27(12)$ & \\
\hline $\begin{array}{l}\text { Non-U.S., invalid, or } \\
\text { missing }\end{array}$ & $6(0.6)$ & $0(0)$ & $1(0.4)$ & \\
\hline
\end{tabular}

Abbreviations: ED, emergency department; ICU, intensive care unit; IQR, interquartile range; RPM, Remote Patient Monitoring; US, United States. ${ }^{\text {a}}$ Statistics presented: $n(\%)$; median (IQR).

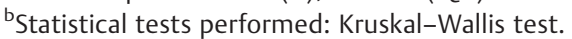

generation processes into routine care, RPM facilitates the functions of a so-called "learning health system." The realtime adaptation that typifies learning health systems is invaluable when dealing with a previously unknown illness, such as COVID-19.

Using technology to remotely monitor patients in their home environment has been of interest for decades across many different diseases, but unfortunately has mixed results from hundreds of randomized trials. ${ }^{39}$ Remote monitoring for congestive heart failure, for example, a common condition leading to hospitalization (and subsequent re-admissions)has had variable results ${ }^{21,40-42}$-as have numerous other conditions. ${ }^{16,43,44}$ There is significant heterogeneity in this space; clinical condition, usage of devices, or wearables and program implementation are just some of the variables that might impact program success. Early evidence, as well as numerous press releases, have indicated a significant interest in using RPM for COVID-19. Our work suggests that RPM can be an effective method of monitoring patients with COVID-19 being discharged from the hospital, that it may lead to a 
Table 2 Remote patient monitoring program patient engagement characteristics

\begin{tabular}{|l|l|}
\hline Characteristics & Value \\
\hline Number of patient days in program & 2,291 \\
\hline Number of questionnaires submitted & 2,161 \\
\hline $\begin{array}{l}\text { Program duration, per patient (day, } \\
\text { median [IQR]) }\end{array}$ & $12(10-13)$ \\
\hline $\begin{array}{l}\text { Number of questionnaire responses, } \\
\text { per patient (median [IQR]) }\end{array}$ & $11(8-13)$ \\
\hline $\begin{array}{l}\text { Patients that completed at least one } \\
\text { survey }\end{array}$ & 210 \\
\hline $\begin{array}{l}\text { Patients that triggered a } \\
\text { symptom/vital sign alert }\end{array}$ & $72 / 210(34 \%)$ \\
\hline $\begin{array}{l}\text { Number of questionnaires triggering } \\
\text { alerts }\end{array}$ & $315 / 2,161(15 \%)$ \\
\hline Number of phone calls to patients & 868 \\
\hline Escalated questionnaires that reported: & $11 / 315(3 \%)$ \\
\hline Oz saturation < $92 \%$ & $239 / 315(76 \%)$ \\
\hline Temperature $>100.3$ & $16 / 315(5 \%)$ \\
\hline Vomiting & $17 / 315(5 \%)$ \\
\hline Worse appetite & $28 / 315(9 \%)$ \\
\hline Worse cough & $27 / 315(9 \%)$ \\
\hline Worse shortness of breath & $31 / 315(10 \%)$ \\
\hline Worse weakness & \\
\hline
\end{tabular}

Abbreviation: IQR, interquartile range. decreased likelihood of presenting to the ED or hospital, and that most patients were monitored without active intervention by our monitoring nurses.

Unfortunately, COVID-19 has disproportionately impacted racial and ethnic minorities, especially African American, Native American, Latinx, and other underserved groups. ${ }^{46-48}$ This was true in our population as well; more than $30 \%$ of our total population with COVID-19 spoke Spanish, for example, which is substantially higher than baseline (in Boston, $14 \%$ of residents speak Spanish at home).$^{49}$ Our program implementation team worked diligently to ensure access to the RPM program to all patients, and our results demonstrated reasonably equitable enrollment, with no statistically significant difference in enrollment between racial or ethnic groups, though there were statistically fewer patients who spoke Spanish that ended up fully enrolled. More broadly, it is important to recognize that requiring a smart phone or desktop computer, having only English and Spanish translations, and relying on provider discretion for referral created the potential for systemic structural inequities in access to the RPM program. We attempted to mitigate these systemic exclusions by enabling proxy access for data entry and 24-hour phone-interpreter support, by using inpatient staff to help with portal access and by explicitly acknowledging concerns around disparities with our team members, but we recognize this may have not been sufficient. Future work should build on this program with an increased focus on health equity concerns.

Our study has several limitations. First, as a five-hospital study, it may not generalize to other hospitals or geographies and we only had access to readmission within our healthcare

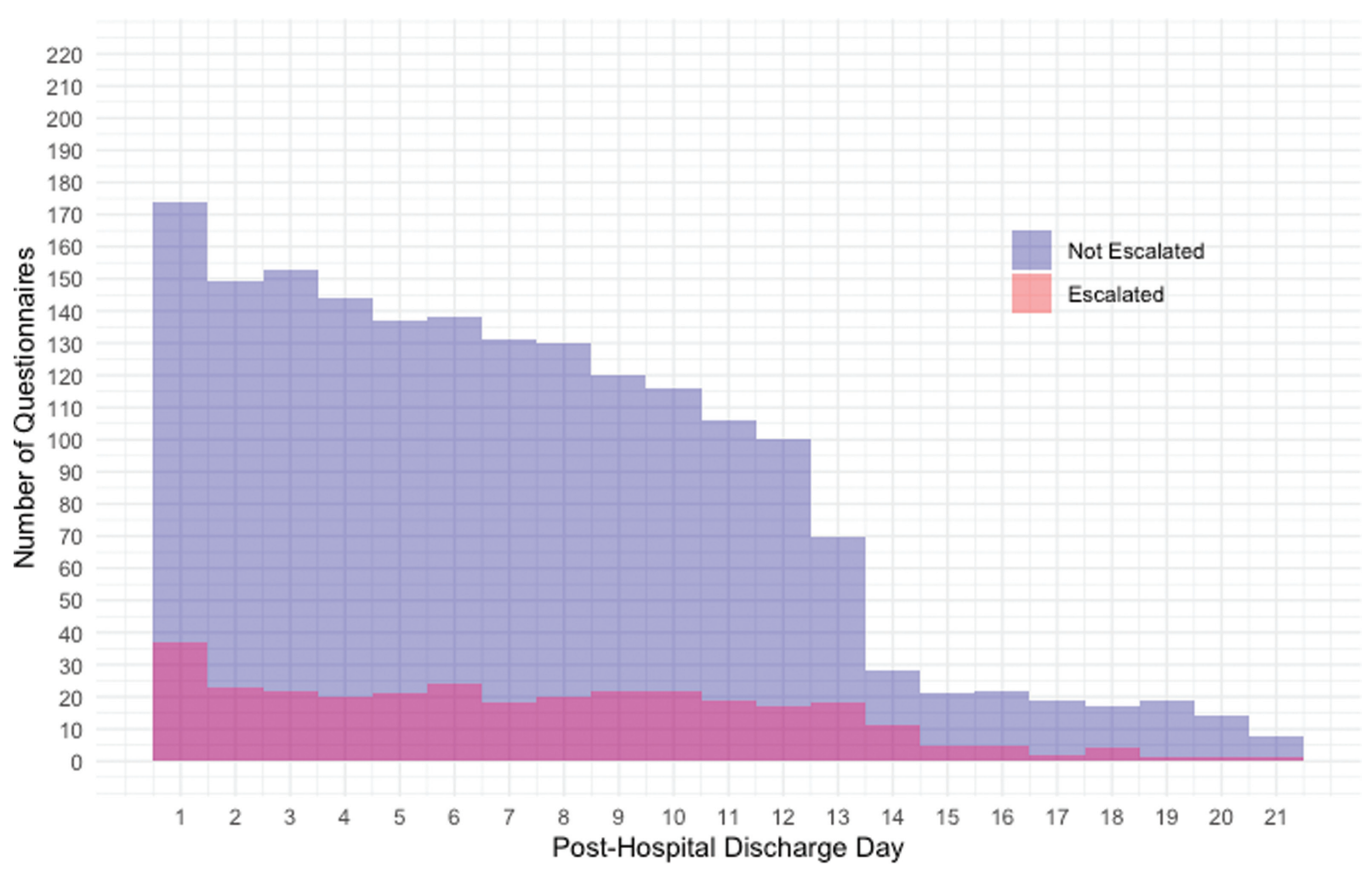

Fig. 3 Frequency of completed questionnaires by post-hospital discharge day. Shown are the frequency of surveys triggering escalation based on post-hospital discharge day for the entire population of enrolled patients. 
Table 3 Mixed-effects adjusted logistic regression model for odds that a patient with COVID-19, initially discharged home, would be readmitted to the hospital or emergency department

\begin{tabular}{|c|c|c|c|c|c|}
\hline \multirow[t]{2}{*}{ Characteristic } & \multicolumn{2}{|c|}{ Unadjusted by readmission status } & \multicolumn{3}{|c|}{ Readmission to ED/hospital } \\
\hline & $\begin{array}{l}\text { Not readmitted, } \\
n=1,227^{\mathrm{a}}\end{array}$ & $\begin{array}{l}\text { Readmitted, } \\
n=129^{\mathrm{a}}\end{array}$ & OR & $95 \% \mathrm{Cl}$ & $p$-Value \\
\hline \multicolumn{6}{|l|}{ Gender (\%) } \\
\hline Female & $616(50)$ & $66(51)$ & & & \\
\hline Male & $611(50)$ & $63(49)$ & 1.04 & $0.72-1.51$ & 0.84 \\
\hline Age & $54(17)$ & $55(17)$ & 0.99 & $0.98-1.01$ & 0.29 \\
\hline \multicolumn{6}{|l|}{ Language (\%) } \\
\hline English & $663(54)$ & $87(67)$ & & & \\
\hline Spanish & $443(36)$ & $32(25)$ & 0.83 & $0.42-1.66$ & 0.60 \\
\hline Not available/other (\%) & $121(9.9)$ & $10(7.8)$ & 0.89 & $0.43-1.84$ & 0.76 \\
\hline \multicolumn{6}{|l|}{ Race (\%) } \\
\hline Black or African American & $215(18)$ & $26(20)$ & & & \\
\hline White & $441(36)$ & $66(51)$ & 1.17 & $0.71-1.95$ & 0.54 \\
\hline Not available /other & $571(47)$ & $37(29)$ & 0.65 & $0.35-1.18$ & 0.16 \\
\hline \multicolumn{6}{|l|}{ Ethnicity (\%) } \\
\hline Hispanic or Latino & $499(41)$ & $39(30)$ & & & \\
\hline Not Hispanic or Latino & $619(50)$ & $86(67)$ & 1.12 & $0.57-2.23$ & 0.74 \\
\hline Other/not available & 109 (8.9) & $4(3.1)$ & 0.34 & $0.11-1.10$ & 0.073 \\
\hline Length of stay & $5.0(3.0-8.0)$ & $4.0(2.0-8.0)$ & 1.02 & $0.99-1.04$ & 0.16 \\
\hline Required ICU & $311(25)$ & $34(26)$ & 0.92 & $0.58-1.47$ & 0.73 \\
\hline \multicolumn{6}{|l|}{ Median income by zip code } \\
\hline $4,836-41,406.5$ & $589(48)$ & $44(34)$ & & & \\
\hline $41,406.5-51,897$ & $325(26)$ & $39(30)$ & 1.45 & $0.90-2.31$ & 0.13 \\
\hline $51,897-65,903.5$ & $191(16)$ & $27(21)$ & 1.60 & $0.93-2.74$ & 0.089 \\
\hline $65,903.5-244,671$ & $116(9.5)$ & $18(14)$ & 1.65 & $0.87-3.13$ & 0.12 \\
\hline Non-U.S., invalid, or missing & $6(0.5)$ & $1(0.8)$ & 2.05 & $0.23-18.2$ & 0.52 \\
\hline \multicolumn{6}{|l|}{ Program status } \\
\hline RPM not ordered & $954(78)$ & $107(83)$ & & & \\
\hline RPM ordered not enrolled & $62(5.1)$ & $8(6.2)$ & 1.11 & $0.51-2.41$ & 0.79 \\
\hline RPM ordered enrolled & $211(17)$ & $14(11)$ & 0.54 & $0.30-0.97$ & 0.039 \\
\hline
\end{tabular}

Abbreviations: ED, emergency department; ICU, intensive care unit; OR, odds ratio; IQR, interquartile range; RPM, Remote Patient Monitoring; SD, standard deviation; US, United States.

a Statistics presented: $n(\%)$; mean (SD); median (IQR).

system. Second, referral into the program was left up to the discretion of the discharging physician, and there is likely uncaptured bias related to which patients were referred to the program; our results do not imply causality. Third, our program required a smart phone or a desktop computer (and internet or a data plan); we did not put other models in place (like telephone only) for this program. Fourth, we did not look at specific associations between responses and readmissions risk (for example, associations between fever and readmission). Finally, we did not look at outcomes beyond readmission, like overall morbidity and mortality associated with RPM. While we would not expect RPM to worsen morbidity and mortality, it is possible that the tendency toward less readmission may have also delayed necessary presentations, leading to worse outcomes.

\section{Conclusion}

We describe the implementation of a COVID-19 RPM program and show that RPM for patients with COVID-19 discharged from one of five hospitals in a single healthcare system was associated with decreased risk of readmission to a combined endpoint of ED or hospital. We also show that a majority of patients enrolled could be passively monitored, which might enable scalable delivery models for COVID-19 in the post-acute setting. Further work is needed to understand 
the financial implications of these types of programs, as well as impact on overall clinical outcomes.

\section{Clinical Relevance Statement}

RPM for patients with COVID-19 being discharged home from the hospital was associated with a decreased risk of readmission to the ED or hospital. Additionally, RPM provided a scalable mechanism to monitor patients in their home environment, with most patients not requiring active intervention from nursing staff.

\section{Multiple Choice Questions}

1. Among patients recovering from COVID-19 at home who reported symptoms or abnormal vital signs through an RPM platform, which abnormality was most common?
a. Temperature
b. Oxygen saturation
c. Worsening shortness of breath
d. Vomiting

Correct Answer: The correct answer is option a. According to our data, for all abnormal surveys completed by patients with COVID-19 discharged from the hospital, the presence of a temperature $>100.3$ was the most common abnormal vital sign or symptom reported. This is an important finding, as it suggests that fever can remain an important symptom of patients with COVID-19 even after they have been hospitalized.

2. Which of the following percentages most accurately describes the percent population of patients with COVID-19 discharged from the hospital that can be monitored passively, that is, without nursing intervention?
a. $36 \%$
b. $46 \%$
c. $56 \%$
d. $66 \%$

Correct Answer: The correct answer is option d. Enrollment in a postdischarge COVID-19 RPM program resulted in many patients completing surveys each day; however, only $34 \%$ of those patients reported abnormal symptoms requiring direct nursing follow-up during their enrollment in the program. This suggests that the majority of patients can be monitored passively, and that an RPM program is a scalable mechanism of providing clinical monitoring for patients with COVID-19 discharged from the hospital.

3. Enrollment in a postdischarge COVID-19 RPM program was associated with which of the following outcomes?
a. Improved 30-day all cause-mortality
b. Decreased combined ED or inpatient readmission
c. Less utilization of primary care resources
d. Improvement in time to being COVID-19 PCR negative

Correct Answer: The correct answer is option b. Enrollment in a postdischarge COVID-19 RPM program was associated with a statistically significant decreased odds of a combined endpoint of re-admission to the hospital or ED. We did not look at mortality of primary care resource utilization as an outcome, and (D) is incorrect as we did not routinely reassess test-status (e.g., PCR positivity) once the patient was discharged from the hospital.

\section{Authors' Contributions}

W.J.G. was involved in article conception. W.J.G., A.D., J.J., M.Y.S., L.M., and D.S. were involved in data collection. W.J. G., D.H., A.D., D.M.L., L.M., D.S., and R.B. were involved in data interpretation and visualization. W.J.G., D.M.L., D.H., and R.B. were involved in statistical analysis. W.J.G. wrote the manuscript draft. All authors were involved in critical revisions, editing, and manuscript approval.

\section{Protection of Human and Animal Subjects}

The study was performed in compliance with the World Medical Association Declaration of Helsinki on Ethical Principles for Medical Research Involving Human Subjects, and as a quality improvement project, the MassGeneral Brigham IRB exempted this study from review.

\section{Conflict of Interest}

W.J.G. reports research funding from IBM, outside the scope of this study. W.J.G. reports consulting income from the Office of the National Coordinator, U.S. Department of Health and Human Services, outside the scope of this study. D.M.L. reports grants from Biofourmis, outside the submitted work.

\section{References}

1 World Health Organization. WHO coronavirus disease (COVID19) dashboard. Available at: https://COVID19.who.int/. Accessed July 20, 2020

2 Helmy YA, Fawzy M, Elaswad A, Sobieh A, Kenney SP, Shehata AA. The COVID-19 pandemic: a comprehensive review of taxonomy, genetics, epidemiology, diagnosis, treatment, and control. J Clin Med 2020;9(04):E1225

3 Chen N, Zhou M, Dong X, et al. Epidemiological and clinical characteristics of 99 cases of 2019 novel coronavirus pneumonia in Wuhan, China: a descriptive study. Lancet 2020;395(10223):507-513

4 White DB, Lo B. A framework for rationing ventilators and critical care beds during the COVID-19 pandemic. JAMA 2020;323(18): 1773-1774

5 Kliff S. Hospitals knew how to make money. Then coronavirus happened The New York Times. Available at: https://www. nytimes.com/2020/05/15/us/hospitals-revenue-coronavirus. html. Published May 15, 2020. Accessed July 20, 2020

6 Reuters. California hospitals struggle financially after preparing for COVID-19 surge that never came. The New York Times. Available at: https://www.nytimes.com/reuters/2020/05/28/us/ 28reuters-health-coronavirus-california-hospitals.html. Published May 28, 2020 Accessed July 20, 2020

7 Khullar D, Bond AM, Schpero WL. COVID-19 and the financial health of US hospitals. JAMA 2020;323(21):2127-2128

8 Peters AW, Chawla KS, Turnbull ZA. Transforming ORs into ICUs. N Engl J Med 2020;382(19):e52

9 Grasselli G, Pesenti A, Cecconi M. Critical care utilization for the COVID-19 outbreak in Lombardy, Italy: early experience and forecast during an emergency response. JAMA 2020;323(16):1545-1546 
10 Webster P. Virtual health care in the era of COVID-19. Lancet 2020;395(10231):1180-1181

11 Dorsey ER, Topol EJ. State of telehealth. N Engl J Med 2016;375 (02):154-161

12 Schwamm LH, Erskine A, Licurse A. A digital embrace to blunt the curve of COVID19 pandemic. NPJ Digit Med 2020;3(01):64

13 Hollander JE, Carr BG. Virtually perfect? Telemedicine for COVID19. N Engl J Med 2020;382(18):1679-1681

14 Shachar C, Engel J, Elwyn G. Implications for telehealth in a postpandemic future: regulatory and privacy issues. JAMA 2020;323(23):2375-2376

15 Annis T, Pleasants S, Hultman G, et al. Rapid Implementation of a COVID-19 Remote Patient Monitoring Program. J Am Med Inform Assoc JAMIA Published online May 11, 2020. Doi: 10.1093/jamia/ ocaa097

16 Noah B, Keller MS, Mosadeghi S, et al. Impact of remote patient monitoring on clinical outcomes: an updated meta-analysis of randomized controlled trials. NPJ Digit Med 2018;1:20172

17 Vegesna A, Tran M, Angelaccio M, Arcona S. Remote patient monitoring via non-invasive digital technologies: a systematic review. Telemed J E Health 2017;23(01):3-17

18 Daley CN, Chen EM, Roebuck AE, et al. Providing patients with implantable cardiac device data through a personal health record: a qualitative study. Appl Clin Inform 2017;8(04):1106-1116

19 Ahmed R, Toscos T, Rohani Ghahari R, et al. Visualization of cardiac implantable electronic device data for older adults using participatory design. Appl Clin Inform 2019;10(04):707-718

20 Fisher NDL, Fera LE, Dunning JR, et al. Development of an entirely remote, non-physician led hypertension management program. Clin Cardiol 2019;42(02):285-291

21 Koehler F, Winkler S, Schieber M, et al; Telemedical Interventional Monitoring in Heart Failure Investigators. Impact of remote telemedical management on mortality and hospitalizations in ambulatory patients with chronic heart failure: the telemedical interventional monitoring in heart failure study. Circulation 2011;123(17):1873-1880

22 Pevnick JM, Fuller G, Duncan R, Spiegel BMR. A Large-scale initiative inviting patients to share personal fitness tracker data with their providers: initial results. PLoS One 2016;11(11):e0165908

23 Ong MK, Romano PS, Edgington S, et al; Better Effectiveness After Transition-Heart Failure (BEAT-HF) Research Group. Effectiveness of remote patient monitoring after discharge of hospitalized patients with heart failure: the better effectiveness after transition - heart failure (BEAT-HF) randomized clinical trial. JAMA Intern Med 2016;176(03):310-318

24 Wallace EL, Rosner MH, Alscher MD, et al. Remote patient management for home dialysis patients. Kidney Int Rep 2017;2 (06):1009-1017

25 Lee PA, Greenfield G, Pappas Y. The impact of telehealth remote patient monitoring on glycemic control in type 2 diabetes: a systematic review and meta-analysis of systematic reviews of randomised controlled trials. BMC Health Serv Res 2018;18(01):495

26 Tomasic I, Tomasic N, Trobec R, Krpan M, Kelava T. Continuous remote monitoring of COPD patients-justification and explanation of the requirements and a survey of the available technologies. Med Biol Eng Comput 2018;56(04):547-569

27 Docherty AB, Harrison EM, Green CA, et al; ISARIC4C investigators. Features of 20133 UK patients in hospital with COVID-19 using the ISARIC WHO Clinical Characterisation Protocol: prospective observational cohort study. BMJ 2020;369:m19

28 Richardson S, Hirsch JS, Narasimhan M, et al; the Northwell COVID19 Research Consortium. Presenting Characteristics, Comorbidities, and Outcomes Among 5700 Patients Hospitalized With COVID-19 in the New York City Area. JAMA 2020;323(20):2052-2059

29 Goyal P, Choi JJ, Pinheiro LC, et al. Clinical characteristics of COVID19 in New York City. N Engl J Med 2020;382(24):2372-2374

30 Medina M, Babiuch C, Card M, et al. Home monitoring for COVID19. Cleve Clin J Med 2020. Doi: 10.3949/ccjm.87a.ccc028
31 Sinai MHealth System. Mount Sinai uses remote patient monitoring to rapidly respond to COVID-19. Published April 6, 2020. Available at: https://www.newswise.com/coronavirus/mountsinai-uses-remote-patient-monitoring-to-rapidly-respond-toCOVID-19/?article_id=729377. Accessed July 20, 2020

32 Landi H. Providence St. Joseph using Twistle remote monitoring technology for 700 COVID patients. Published April 1, 2020. Available at: https://www.fiercehealthcare.com/tech/providence-using-twistle-remote-monitoring-technology-for-700COVID-patients. Accessed July 20, 2020

33 Park PG, Kim CH, Heo Y, Kim TS, Park CW, Kim CH. Out-of-hospital cohort treatment of coronavirus disease 2019 patients with mild symptoms in Korea: an experience from a single community treatment center. J Korean Med Sci 2020;35(13):e140

34 Grutters LA, Majoor KI, Mattern ESK, Hardeman JA, van Swol CFP, Vorselaars ADM. Home telemonitoring makes early hospital discharge of COVID-19 patients possible.J Am Med Inform Assoc JAMIA Published online July 15, 2020. Doi: $10.1093 /$ jamia/ocaa168

35 Hron JD, Parsons CR, Williams LA, Harper MB, Bourgeois FC. Rapid implementation of an inpatient telehealth program during the COVID-19 pandemic. Appl Clin Inform 2020;11(03):452-459

36 Grange ES, Neil EJ, Stoffel M, et al. Responding to COVID-19: The UW medicine information technology services experience. Appl Clin Inform 2020;11(02):265-275

37 Massachusetts. COVID-19 Response reporting. Published June 8, 2020. Available at: https://www.mass.gov/info-details/COVID19-response-reporting. Accessed July 20, 2020

38 Chen J, Qi T, Liu L, et al. Clinical progression of patients with COVID-19 in Shanghai, China. J Infect 2020;80(05):e1-e6

39 Wootton R. Twenty years of telemedicine in chronic disease management-an evidence synthesis. J Telemed Telecare 2012; 18(04):211-220

40 Chaudhry SI, Mattera JA, Curtis JP, et al. Telemonitoring in patients with heart failure. N Engl J Med 2010;363(24):2301-2309

41 Bashi N, Karunanithi M, Fatehi F, Ding H, Walters D. Remote monitoring of patients with heart failure: an overview of systematic reviews. J Med Internet Res 2017;19(01):e18

42 Inglis SC, Clark RA, McAlister FA, Stewart S, Cleland JGF. Which components of heart failure programmes are effective? A systematic review and meta-analysis of the outcomes of structured telephone support or telemonitoring as the primary component of chronic heart failure management in 8323 patients: Abridged Cochrane Review. Eur J Heart Fail 2011;13(09):1028-1040

43 Hameed AS, Sauermann S, Schreier G. The impact of adherence on costs and effectiveness of telemedical patient management in heart failure: a systematic review. Appl Clin Inform 2014;5(03): 612-620

44 Ralston JD, Cook AJ, Anderson ML, et al. Home blood pressure monitoring, secure electronic messaging and medication intensification for improving hypertension control: a mediation analysis. Appl Clin Inform 2014;5(01):232-248

45 Kricke G, Roemer PE, Barnard C, et al. Rapid implementation of an outpatient COVID-19 monitoring program. NEJM Catal Innov Care Deliv. Published online June 16, 2020. Available at: https://catalyst. nejm.org/doi/abs/10.1056/cat.20.0214. Accessed June 28, 2020

46 Price-Haywood EG, Burton J, Fort D, Seoane L. Hospitalization and mortality among black patients and white patients with COVID19. N Engl J Med 2020;382(26):2534-2543

47 Tai DBG, Shah A, Doubeni CA, Sia IG, Wieland ML. The disproportionate impact of COVID-19 on racial and ethnic minorities in the United States. Clin Infect Dis Off Publ Infect Dis Soc Am. Published online June 20, 2020. Doi: 10.1093/cid/ciaa815

48 Greenaway C, Hargreaves S, Barkati S, et al. COVID-19: exposing and addressing health disparities among ethnic minorities and migrants. J Travel Med 2020:taaa113. Doi: 10.1093/jtm/taaa113

49 Jimenez CR. New Bostonians demographic report. Available at: https://www.cityofboston.gov/newbostonians/pdfs/dem_report. pdf. Accessed September 11, 2020 